\title{
Evidence-based Teaching with the Help of Mobile Response System (MRS)
}

\author{
Mohammad Muztaba Fuad \\ Department of Computer Science \\ Winston-Salem State University \\ Winston-Salem, NC 27110, USA \\ +1-3367503325 \\ fuadmo@wssu.edu
}

\author{
Debzani Deb \\ Department of Computer Science \\ Winston-Salem State University \\ Winston-Salem, NC 27110, USA \\ +1-3367502496 \\ debd@wssu.edu
}

\begin{abstract}
Over the past couple of years, evidence-based teaching and learning methods are brought into focus from the experience gained in clinical psychology and their use of Evidence-Based Practices. Different authors have discussed the advantages of using such evidence-based methods for teaching and learning in academia. Measuring real-time impact of traditional pedagogical approaches used in STEM disciplines are not easy and do not provide faculty an instant evidence about student learning. This paper will present Mobile Response System (MRS) software, which facilitate anonymous communication, interaction and evaluation of in-class interactive problem solving activities using mobile devices. MRS facilitates a feedback-driven and evidencebased teaching methodology, which is important to enhance student learning.
\end{abstract}

\section{CCS Concepts}

-Applied computing $\rightarrow$ Computer-assisted instruction •Applied computing $\rightarrow$ Interactive learning environments

\section{Keywords}

Interactive problem solving; mobile technology; active learning.

\section{INTRODUCTION}

Active learning is an educational approach where learners become engaged in interactive learning activities in the classroom. Studies [1]-[2] reveal strong empirical evidence that active involvement in the learning process is crucially important for the mastery of skills, such as critical thinking. Specifically in STEM disciplines, it is important for learners to actively study a problem and explore possible solutions by interacting with the problem in a hands-on approach. In such situations, traditional pedagogical approaches are not enough and more interactive teaching and learning strategy is necessary to make learning more productive. This research explores whether by presenting the in-class exercises as visual interactive entities (where students can actively play with the problem), student's critical thinking and problem solving

\footnotetext{
Permission to make digital or hard copies of part or all of this work for personal or classroom use is granted without fee provided that copies are not made or distributed for profit or commercial advantage and that copies bear this notice and the full citation on the first page. Copyrights for third-party components of this work must be honored. For all other uses, contact the Owner/Author.

Copyright is held by the owner/author(s).

ITiCSE '16, July 09-13, 2016, Arequipa, Peru

ACM 978-1-4503-4231-5/16/07.

http://dx.doi.org/10.1145/2899415.2925498
}

skills can be improved. In recent years, mobile technology has brought incredible opportunities for educators to enable and deliver learning in ways that could not have been accomplished before. There have been an increasing number of studies [3-4] related to the research and development of learning software intended for mobile computing devices. By having interactive mobile App quizzes rather than traditional pen-and-paper quizzes, the goal of this research is to make the quizzes more appealing to students and to allow them to realize the effect of their interactions at the different stage of the exercise. The presented approach allows the student to fully comprehend a concept and clarify any confusion through active interaction and hands-on nature of the system. This also allows students to practice the concept outside the class using these interactive quiz applications and allows faculty to administer more of it in class without the stress of manual grading.

\section{Mobile Response System}

This paper presents the Mobile Response System (MRS) [5] software, which facilitate anonymous communication, interaction and evaluation of in-class interactive problem solving activities using mobile devices. MRS facilitates a feedback-driven and evidence-based teaching methodology, which is important to enhance student learning. By allowing interactive problem solving using mobile devices and by being extensible to other disciplines, MRS makes itself distinctive than similar systems. MRS is a client-server software that allows the faculty to dynamically prompt the students with interactive exercises synchronized with the lecture material in their mobile devices. Students are able to actively interact with the problem and send their answers back to the faculty computer. MRS then grades the exercise by comparing the student made sequence of steps with the correct sequence of steps. After grading, MRS also makes the grading statistics and student submissions available for the faculty to view and share with the students.

This formative assessment information allows the faculty to have real-time evidence of students' comprehension of covered lecture materials on a particular class and also helps faculty to identify the concepts that need to be repeated or reinforced. By utilizing MRS software, faculty is also able to capture screens from student submissions and discuss on those screens if context-sensitive feedback is needed. Additionally, this approach allows the students to obtain faster and frequent feedback that reinforce their learning and help them to identify problem areas. The active interaction with a problem via multiple steps while going back and forth and seeing the consequence of their choices at each step is expected to enhance students' analytical and problem solving 
capabilities. The other important feature supported by MRS is the ability to submit feedback/question anonymously during the class. The software allows students to send anonymous feedback/ questions to faculty and vote on feedback/questions that faculty will choose to review and answer at the end of the class.

\subsection{Software Architecture}

The MRS software is designed as a client-server application as shown in Figure 1. The faculty computer runs the server component of the software, which hosts questions, manages users and maintain communication and synchronization. The client component executes in student's mobile device, which allows students to login to the system, to submit anonymous feedback/questions and facilitates interactive exercise solving.

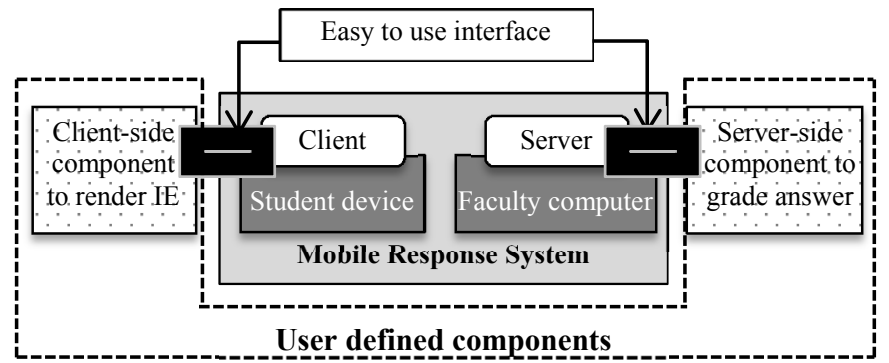

Figure 1. MRS Software Architecture

Building each possible problem type within the MRS client is impossible and will make it domain dependent and overly bulky to run on mobile platforms. To overcome this, the interactive activity is designed to be separated from the application logic of the MRS client software. Similarly on the server side, the grading component is also separated from the application logic, so that faculty can use any statistical or grading instrument on the student responses. Both the server and the client provides easy to use application programmer interface for users to develop their own interactive problem solving apps and corresponding grading modules to be used within MRS environment.

\subsection{Interactive Problems}

An Interactive Problem in MRS allows students to devise the answer by following a set of steps and by using a particular algorithm/process. In each step, students have to make key choices that will have impact on the next step of the interaction. During these interaction steps, students can go back and forth and change their answer. This will allow them to see what is the effect of different selection on the result and how every piece fits together. Problems can be started bottom up or at the middle to give students different perspective on the problem and assess their problem solving skills. Only after the student traverses each of the steps or the allotted time to answer a problem runs out, the result of their interaction is sent back to the server as the answer of that student. The rubric for each problem supports partial grading along with grading correct answers.

\subsection{MRS at work}

MRS environment provides an intuitive and easy to use interface for learners and instructors to utilize the software. The faculty can use server-side user interface (Figure 2(a)) to import student information, initiate the system, import questions, broadcast question to students, process results, monitor student's feedback/question etc. On the client-side, student can use the client interface (Figure 2(b)) to login to the system, submit anonymous feedback/question or vote on an existing feedback /question. Once the client receives a question, it locates the app that will render the question into interactive activity. After student submit their answer, the client sends it back to the faculty computer for result processing.

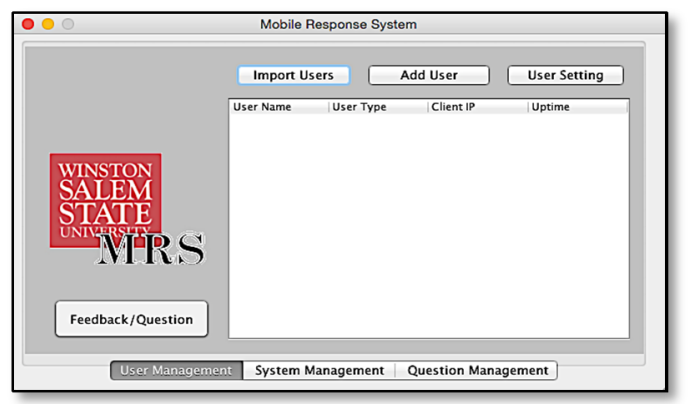

(a)

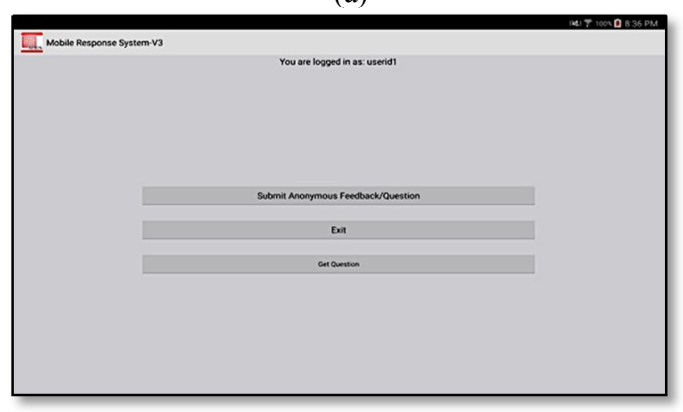

(b)

Figure 2. MRS User Interfaces.

\subsection{Current status}

Currently, the developed MRS software and corresponding course modules are being deployed in one sophomore and one junior Computer Science course. The data obtained from the survey, focus group and student grade distribution supports the effectiveness of the software. For both intervened classes, students positively attested that MRS and related apps helped them to learn through hands-on activities that they enjoyed.

\section{ACKNOWLEDGMENTS}

This research is supported by National Science Foundation grant \#1332531.

\section{REFERENCES}

[1] J. M. Braxton, W. A. Jones, A. S. Hirschy, \& H. V. Hartley, The role of active learning in college persistence. New Directions for Teaching and Learning, 115:71-83, 2008.

[2] M. Prince, Does active learning work? A review of the research. Journal of Engineering Education, 93(3): 223231, 2004.

[3] R. J. Young, Mobile College App: Turning iPhones Into 'Super-Clickers' for Classroom Feedback, Chronicle of higher education, 2008.

[4] K. Whattananarong, An Experiment in the Use of Mobile Phones for Testing at King Mongkut's Institute of Technology North Bangkok, Thailand. Proc. International Conference on Making Education Reform Happen: Learning from the Asian Experience \& Comparative Perspectives, 2004.

[5] Mobile Response System, http://compsci.wssu.edu/MRS. 